5 Abstract

\title{
An interactive web-based geovisual analytics platform for co-clustering
}

\section{spatio-temporal data ${ }^{1}$}

\author{
Xiaojing Wu*, Ate Poorthuis, Raul Zurita-Milla, Menno-Jan Kraak \\ *corresponding author. Email: 11132018618@bnu.edu.cn
}

Clustering methods are useful in analyzing patterns from big spatio-temporal data. However, previous studies typically rely on traditional clustering methods to explore spatial or temporal patterns. Coclustering methods allow the concurrent analysis of spatial and temporal patterns by identifying locationand timestamp-clusters at the same time. By combining co-clustering with coordinated multiple views (CMV) in an interactive geovisual analytics platform, we facilitate the exploratory co-clustering analysis of spatio-temporal data and the results. Further enhanced by Web 2.0 standards, our geovisual analytics platform ease the access to co-clustering analysis from any web browser. More specifically, our platform allows users to upload data and to visually explore it using interactive CMV to help the selection of coclustering parameters. Our platform also allows users to run co-clustering and to visually and interactively explore the results. To illustrate the use of our platform, we analyze Dutch annual average temperature for 28 stations from 1992 to 2011 . Results show that our platform not only helps to get a better understanding of the dataset but also to choose the co-clustering parameters. Our platform helps to interpret the coclustering results too, and it supports the extraction and exploration of complex patterns buried in the data. In the era of big data, our web-based platform enables the exploration of concurrent spatio-temporal patterns from large datasets by combing both computer power and human interpretative capabilities.

Keywords: web-based; geovisual analytics; interactive coordinated multiple views; clustering analysis; spatio-temporal pattern

\footnotetext{
${ }^{1}$ Xiaojing Wu: developed platform and wrote manuscript. Ate Poorthuis: gave advices on platform and contributed to the manuscript. Raul Zurita-Milla: gave advices regarding the algorithm and contributed to the final manuscript. Menno-Jan Kraak: reviewed the final version of the manuscript
} 


\section{Introduction}

Thanks to the advancement in data collection technologies, the amount of spatio-temporal data have

25 dramatically increased, and the extraction of knowledge from these data becomes a challenge [1, 2]. As

26 one of data mining tasks, clustering methods are very useful in analyzing patterns and further extracting

27 the underlying knowledge. Most previous studies on clustering analysis of spatio-temporal data reply on

28 one-way clustering, also called traditional clustering (Figure 1), which analyzes the data from spatial or

29 temporal aspect separately. Specifically, spatial clustering groups locations in the data into clusters with

30 similar values of the attribute(s) along all timestamps (Figure 1b) whereas temporal clustering groups

31 timestamps into clusters with similar value along all locations (Figure 1c)[3, 4]. However, such clustering

32 analysis only consider the space- or time-varying behavior of the data [5].

33

\begin{tabular}{|c|c|c|c|}
\hline $\begin{array}{c}\text { Spatio-temporal } \\
\text { data }\end{array}$ & timestamp-1 & timestamp-2 & timestamp-3 \\
\hline location-1 & 4.2 & 5.0 & 4.9 \\
\hline location-2 & 5.7 & 3.5 & 3.4 \\
\hline \multirow[t]{2}{*}{ location-3 } & 5.5 & 3.6 & 3.5 \\
\hline & temporal & \multicolumn{2}{|l|}{ clustering } \\
\hline (c) & $\begin{array}{l}\text { timestamp- } \\
\text { cluster1 }\end{array}$ & \multicolumn{2}{|c|}{ timestamp-cluster2 } \\
\hline $\begin{array}{c}\text { Spatio-temporal } \\
\text { data }\end{array}$ & timestamp-1 & timestamp-2 & timestamp-3 \\
\hline location-1 & 4.2 & 5.0 & 4.9 \\
\hline location-2 & 5.7 & 3.5 & 3.4 \\
\hline location-3 & 5.5 & 3.6 & 3.5 \\
\hline
\end{tabular}

\begin{tabular}{|c|c|c|c|c|c|}
\cline { 3 - 5 } \multicolumn{1}{c|}{$(\mathrm{b})$} & $\begin{array}{c}\text { Spatio-temporal } \\
\text { data }\end{array}$ & timestamp-1 & timestamp-2 & timestamp-3 \\
\cline { 2 - 6 } spatial & $\begin{array}{c}\text { location- } \\
\text { cluster1 }\end{array}$ & location-1 & 4.2 & 5.0 & 4.9 \\
\cline { 2 - 6 } & \multirow{2}{*}{$\begin{array}{c}\text { location- } \\
\text { cluster2 }\end{array}$} & location-2 & 5.7 & 3.5 & 3.4 \\
\cline { 3 - 6 } & location-3 & 5.5 & 3.6 & 3.5 \\
\hline
\end{tabular}

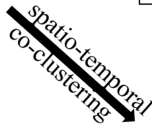

\begin{tabular}{|c|c|c|c|c|}
\cline { 3 - 5 } \multicolumn{2}{c|}{} & $\begin{array}{c}\text { timestamp- } \\
\text { cluster1 }\end{array}$ & \multicolumn{2}{c|}{ timestamp-cluster2 } \\
\cline { 2 - 5 } & $\begin{array}{c}\text { Spatio-temporal } \\
\text { data }\end{array}$ & timestamp-1 & timestamp-2 & timestamp-3 \\
\hline $\begin{array}{c}\text { location- } \\
\text { cluster1 }\end{array}$ & location-1 & 4.2 & 5.0 & 4.9 \\
\hline \multirow{2}{*}{$\begin{array}{c}\text { location- } \\
\text { cluster2 }\end{array}$} & location-2 & 5.7 & 3.5 & 3.4 \\
\cline { 2 - 5 } & location-3 & 5.5 & 3.6 & 3.5 \\
\hline
\end{tabular}

Figure 1: A simulated dataset (a) for spatial clustering (b), temporal clustering (c) and coclustering (d) analysis of spatio-temporal data

Unlike traditional clustering, co-clustering concurrently groups locations to location-clusters and timestamps to timestamp-clusters and identifies co-clusters where values of the attribute(s) are similar along both locations and timestamps (Figure 1d). As such, co-clustering analysis considers both space and time-varying behavior in the data. Having been used in various fields [6-8], co-clustering methods attract 
attentions in geo-field recently [9-11], where Bregman block average co-clustering algorithm with I-

41 divergence (BBAC_I) is used for the exploration of patterns along both space and time dimensions.

42 However, one common issue for clustering/co-clustering analysis is the selection of parameters, e.g.

43 number of clusters $[12,13]$. These parameters are often predefined by trial and error, expert knowledge or

44 by considering the characteristics of the dataset at hand and/or the application. An exploratory analysis of

45 spatio-temporal data helps to "get acquainted with the data" and to decide on the most suitable parameters

46 for a given problem [14]. This analysis is often steered by user's interactions and visual analysis of the

47 resulting clusters [15].

Various geovisualizations have been used to visually analyze the clustering results in spatio-temporal data. For instance, $\mathrm{Wu}$, Zurita-Milla [3] applied the geographical maps to represent the spatial distribution 50 of spatial clusters in weather data. van den Elzen and van Wijk [16] used small multiples to visually

51 explore clusters in winter holiday accommodations. Storme, Derudder [17] applied cluster heatmaps to 52 visually analyze the complex patterns of interactions between producer services firms and world cities.

As a core functionality of visual analytics, the Coordinated Multiple Views (CMV) provides various

54 linked geovisualizations for different aspects of spatio-temporal data to help its exploration [18]. By integrating CMV, human interactions and computational methods, e.g. clustering/co-clustering, into interactive visual interfaces, geovisual analytics enables an iterative and exploratory analysis with human

57 involved [19]. For instance, Guo and Jiang [20] presented an interactive geovisual analytics approach by

58 integrating multivariate geographical map, parallel coordinates plot (PCP) and self-organizing map to 59 analyze complex crime patterns. Tatiana, Felix [21] developed a geovisual analytics platform by 60 integrating flow map, calendar map and a spatial graph clustering algorithm to analyze movement data.

61 However, previous studies on developing geovisual analytics approaches only used traditional clustering 62 methods for analyzing spatio-temporal data. 
The introduction of Web 2.0 standards enables users to access complex analytical tools and facilitates

the analysis of spatio-temporal data for especially non-experts $[22,23]$. Now with any web browser, users can explore their data using computational methods and visualizations [24]. For example, Sun, Li [25] proposed an interactive web-based geovisual analytics system using statistical analysis and multiple views for climate studies. Liao, Peng [26] developed a web-based geovisual analytics system with CMV to analyze air quality data. However, until now no web-based geovisual analytics platform has been developed for co-clustering spatio-temporal data.

Thus, in this paper, we follow the work of [9] and build an interactive web-based geovisual analytics platform with BBAC_I, which provides users with easy access to the iterative, visual and exploratory coclustering analysis of spatio-temporal data. The structure of this paper is as follows. The co-clustering algorithm (BBAC_I) is explained in section 2 while the developed platform is described in section 3. Then the use of the platform is demonstrated using a case study in section 4 , followed by discussion in section 5 and conclusion in section 6 .

\section{Bregman block average co-clustering algorithm with I-divergence (BBAC_I)}

BBAC_I allows the analysis of any 2-D data matrix with positive real values. Many spatio-temporal datasets can be organized as a co-occurrence matrix $O_{L T}$ between the location and the timestamp variables that take values across $m$ locations and $n$ timestamps. Correspondingly, the co-clustered spatiotemporal data matrix can be represented as the matrix $O_{\hat{L} \hat{T}}$, which contains the location- and timestampcluster variables that take values across $k$ location- and $h$ timestamp-clusters. Rooted in information theory where the mutual information is defined as the quantity of shared information between variables [27], BBAC_I measures the loss of mutual information between $O_{L T}$ and $O_{\hat{L} \hat{T}}$ with information divergence (I-divergence), and minimizes this loss to obtain the optimal co-clustering results. In the following we summarize the main 5 steps of this process (Figure 2) and elaborate on its technical implementation: 


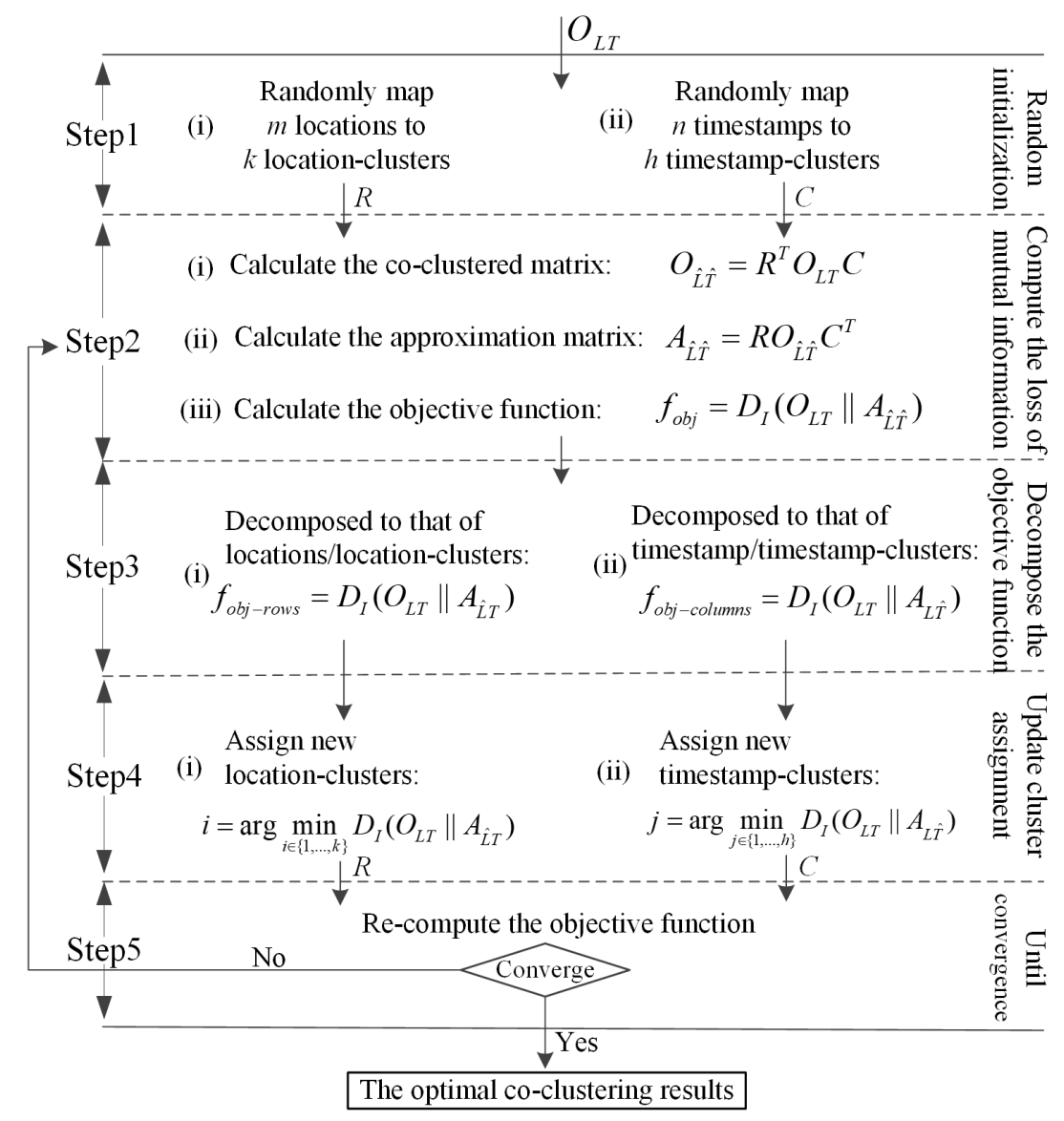

89 (1) Step 1: Perform a random initialization of the clusters. This step is implemented in two operations:

(i) Randomly map $m$ locations to $k$ location-clusters and construct a binary $m \times k$ matrix, $R$, to indicate location-cluster membership;

(ii) Randomly map $n$ timestamps to $h$ timestamp-clusters and construct a binary $n \times h$ matrix, $C$, to indicate timestamp-cluster membership.

(2) Step 2: Compute the loss of mutual information between $O_{L T}$ and $O_{\hat{L} \hat{T}}$. This step is implemented in three operations: in previous step: 
Where $R^{T}$ is the transpose of $R$.

101

(ii) Then calculate the approximation matrix $A_{\hat{L} \hat{T}}$ using $O_{\hat{L} \hat{T}}, R$ and $C$. This steps is needed because $O_{\hat{L} \hat{T}}(k \times h)$ is of different size than $O_{L T}(m \times n)$. Hence, $A_{\hat{L} \hat{T}}$ approximates $O_{\hat{L} \hat{T}}$ but has the same size as $O_{L T}$ :

$$
A_{\hat{L} \hat{T}}=R O_{\hat{L} \hat{T}} C^{T}
$$

105

Where $C^{T}$ is the transpose of $C$. During this operation, BBAC_I preserves the averages of each co-cluster (hence the name block average) along both locations and timestamps.

(iii) Calculate the loss in mutual information between $A_{\hat{L} \hat{T}}$ and $O_{L T}$ with I-divergence, which is the objective function of BBAC_I:

$$
f_{o b j}=D_{I}\left(O_{L T} \| A_{\hat{L} \hat{T}}\right)
$$

Where $D_{I}(\cdot \| \cdot)$ is the I-divergence among two matrices.

(3) Step 3: Decompose the objective function. Equation (3) can be decomposed to that of rows/locations and columns/timestamps [27]. This step is implemented in two operations.

(i) The objective function is decomposed into the I-divergence related to locations and locationclusters:

$$
f_{\text {obj-rows }}=D_{I}\left(O_{L T} \| A_{\hat{L} T}\right)
$$

Which produces a distance matrix of locations/location-clusters with size $m \mathrm{x} k$, indicating the I-divergence metrics of assigning each of $m$ locations to $k$ location-clusters. 
(ii) The objective function is decomposed into the I-divergence related to timestamps and timestamp-clusters:

$$
f_{\text {obj-columns }}=D_{I}\left(O_{L T} \| A_{L \hat{T}}\right)
$$
the I-divergence metrics of assigning each of $n$ locations to $h$ location-clusters.

(4) Step 4: Update cluster assignment. Following step 3, this step is also implemented in two operations.

(ii) Assign new timestamp-clusters. For each of $n$ timestamps, choose the $j^{\text {th }}$ timestamp-cluster which has the minimum I-divergence metric in the distance matrix of timestamps/timestampclusters:

$$
i=\arg \min _{i \in\{1, \ldots, k\}} D_{I}\left(O_{L T} \| A_{\hat{L} T}\right)
$$

(i) Assign new location-clusters. For each of $m$ locations, choose the $i^{\text {th }}$ location-cluster which has the minimum I-divergence metric in the distance matrix of locations/location-clusters: 
After each iteration the objective function decreases monotonically [6], thus BBAC_I can achieve a local convergence. Nevertheless, to optimize the likelihood of achieving a global convergence, the above

141 process is repeated with various initial random mapping (outer iterations). The initialization leading to a 142 smaller loss of mutual information is chosen as the final one. For detailed explanation of BBAC_I we 143 refer the readers to the work of [9] and Banerjee, Dhillon [6].

\section{The web-based geovisual analytics platform}

To facilitate the interactive exploration, our web-based platform consists of a front-end graphical user interface (GUI) and a back-end server (left of Figure 3). Being mainly responsible for the visual views (CMV). While the server side is responsible for the implementation of BBAC_I.

Interactive web-based geovisual analytics platform for co-clustering spatio-temporal data

Front-end Graphical User Interface (GUI)

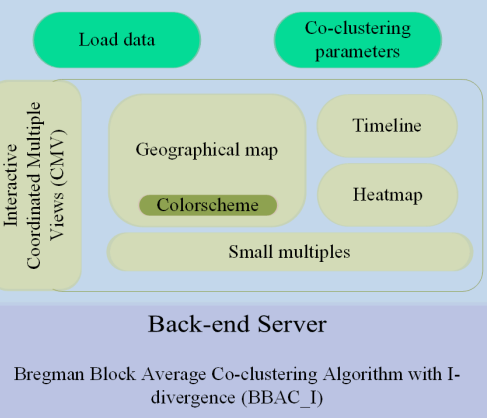

Workflow for co-clustering analysis of spatiotemporal data using the platform

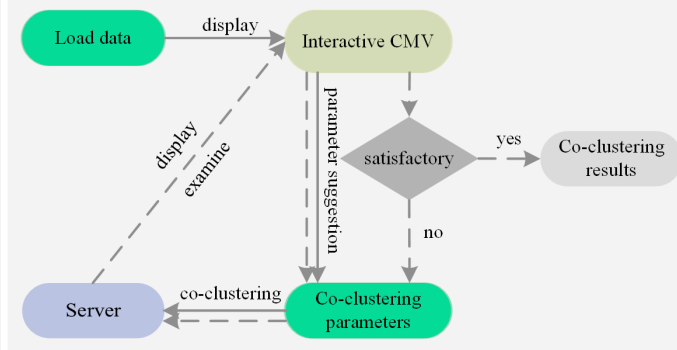

Figure 3: The framework of the interactive web-based geovisual analytics platform (left) and the workflow for analyzing spatio-temporal data (right) where solid arrows relate with loaded data while dashed arrows with co-clustering results letting users upload their own data, both attribute (.csv) and map data (.geojson), which is displayed instantly using CMV. Then they can explore it interactively, which helps to choose the co-clustering parameters (numbers of location-cluster, timestamp-clusters, outer iterations and inner iterations). After 
parameters are selected, GUI will send request to the server with the uploaded data and chosen parameters to ask for being processed with the co-clustering algorithm. When receiving request from the client side

159 (GUI), the server side processes the data using BBAC_I with chosen parameters and afterwards sends the co-clustering results back to GUI for display. Then users can examine the results by visually exploring them. They can also choose different sets of parameters and redo the co-clustering analysis until

162 satisfactory co-clustering results are obtained. As such, this platform can promote users' interactions by 163 allowing them to explore the uploaded data and then discuss their findings on their own.

\subsection{Coordinated multiple views (CMV)} CMV are used, which includes the geographical map, linear timeline, heatmap, small multiples and of them the last one is especially for the co-clustering results.

\subsubsection{Geographical map}

The geographical map is any map from the map data provided by the user. The map is used to visualize the spatial aspects of the original data and co-clustering results. For both cases, it is colored to show the spatial variations of the attribute's value.

The selection of a suitable color scheme is essential. Take the temperature data for example, typically the diverging color scheme $<$ RedYellowBlue $>$ is selected to represent low temperatures with blue and high ones with red [28]. The platform allows users to choose one from a series of diverging and sequential color schemes, which is used for all visualizations.

\subsubsection{Linear timeline}

The linear timeline is used to visualize the temporal aspects of the original data and the co-clustering results which are related to linear timestamps (e.g. years). For both cases, the linear timeline arranges all timestamps sequentially. For the original data, the event of each timestamp is the value of the attribute and the linear timeline shows the temporal variations of the original data. Whereas for co-clustering results, the event of each timestamp indicates 
which timestamp-cluster it belongs to, with timestamp-clusters arranged in y-axis with increasing attribute's value from the first to the last. As such, the liner timeline shows the temporal patterns in the co-clustering results. A toggle button allows users to switch between the two cases for further exploration.

\subsubsection{Heatmap}

Heatmap uses a range of colors to visualize individual values in a data matrix [29]. The heatmap is used here to visualize the attribute aspects of both the original data and the coclustering results. For the former, the heatmap offers an overview of the whole original dataset by arranging timestamps and locations in the $\mathrm{x}$ - and $\mathrm{y}$-axis from the first to last and coloring each record according to its value of the attribute. For the latter, by preserving the axes arrangement but coloring individual records with co-clustered values, the heatmap provides a straightforward view of the co-clustered matrix in the co-clustering results. After the co-clustering process, a toggle button allows users to switch back and forth to make comparisons between the original data matrix and the co-clustered one. Besides, two buttons $(<$ select observe area $>$ and $<$ clear $>)$ allows users to select regions and time intervals of their interest to reveal spatio-temporal patterns in the co-clustering results.

\subsubsection{Small multiples}

Map-based small multiples are a set of adjacent geographical maps to represent changes in relation to timestamps [30]. The small multiples is used here to visualize the co-clustering results. Similar as the above, each map in the small multiples is any map provided by the user. Each map is for each of timestamp-clusters, in which location-clusters are represented with bold boundaries. By this means, the small multiples visualize spatio-temporal coclusters.

\subsection{Interactions using CMV}


To help users gain more insights into both their original data and the co-clustering results, visualizations in CMV are further integrated with the user interactions, e.g. hover-based brushing and linking. That is, a selection made by hover in one visualization will result in changes in other visualizations automatically. Such exploration is facilitated by using spatio-temporal coclustering analysis related tasks [31].

\subsubsection{Exploration of the user's uploaded data}

For the exploration of the original data (the user's uploaded data), questions for tasks respectively. These questions can be answered by user interactions among the geographical map, timeline and heatmap.

The formulated question focusing on the spatial dimension can be 'what is the temporal variation of the attribute in a specified location and which locations have similar variations with a specified location?' To answer it, the user can hover over the specified location in the geographical map, with itself highlighted in the map, the temporal variation of the attribute in this location will be displayed in the timeline and also all the records related to that location will be highlighted in the heatmap. By hovering over other locations in the map, the user can identify locations with similar temporal variations.

The question focusing on the temporal dimension can be 'what is the spatial variation of the attribute in a specified timestamp and which timestamps have similar variations with a specified timestamp?' It can be answered by hovering over the specified timestamp in the timeline, which will result in its highlighting, the changes of colors in the map to show the spatial variations of the attribute's value in that timestamp and also the highlighted records related to that timestamp. Similarly, hovering over other timestamps will help to identify those with similar spatial variations.

The question focusing on the attribute dimension can be 'what is the attribute in the specified location and timestamp?' It can be answered by hovering over the individual record 
in the heatmap specified by a location and a timestamp. With this record highlighted in the heatmap, the spatial location and timestamp of it will be highlighted in the map and timeline separately. As such, these interactions enable users to visually explore their data in detail to

get a better understanding and further help them choose the co-clustering parameters.

\subsubsection{Exploration of co-clustering results}

For the exploration of the co-clustering results, questions for tasks related to the coclustering analysis focus on the individual co-clusters and spatio-temporal patterns respectively, each of them containing subtasks focusing on spatial \& temporal as well as attribute aspect. These questions can be answered by the interactions among the geographical map, timeline, heatmap and small multiples.

Questions focusing on individual co-clusters. The question focusing on the spatial \& temporal aspect of individual co-clusters can be "what is the co-cluster with the specified location and timestamp and what other locations and timestamps are also in that co-cluster?' It can be answered by first hovering over the map, then the timeline and finally the small multiples. When the specified location in the map is hovered over, the corresponding location-cluster will be highlighted in the map and also the small multiples. Then hovering over the specified timestamp in the timeline will highlight the corresponding timestampcluster in the timeline and also the small multiples. The co-cluster for the specified location and timestamp is the intersection of both highlights in the small multiples. Finally, by hovering over the identified co-cluster in the small multiples, the highlighted locations and timestamps in the map and timeline are the ones in the same co-cluster for the specified location and timestamp.

The question focusing on the attribute aspect of individual co-clusters can be 'what are locations and timestamps in a specified co-cluster (e.g. co-cluster with the highest attribute value)?' To answer it, the user can observe and hover over the specified co-cluster in the small multiples. By observing each co-cluster in the small multiples, the user can identify the 
specified one, e.g. with the highest attribute value. Then by hovering over the co-clusters, with itself highlighted in the small multiples, the user can find the locations and timestamps that belonging to this co-cluster highlighted in the map and timeline separately.

Questions focusing on spatio-temporal patterns. Questions for spatio-temporal patterns are more complex and need more interactions to complete the tasks. The formulated question focusing on the spatial \& temporal aspect of spatio-temporal patterns can be "what are the patterns in specified regions and time intervals?' It can be answered by interacting with the heatmap and observing the map and timeline. To select the specified regions, the user can click the labels of two locations in the y-axis of the heatmap, the clicked labels enlarged first and then back to normal to indicate the selection. Similarly, the user can select the specified time intervals by clicking the labels of two timestamps in the x-axis. After the selection, the user can click the $<$ select observe area $>$ button and will see a rectangle appear in the heatmap with the selected regions and intervals. These selections are also highlighted in the map and timeline separately to help users double-check selections and users can always clear the selections using the button $<$ clear $>$ and redo the selection. Finally by observing the records within the rectangle, the user can observe the patterns of the attribute in the specified regions and time intervals.

The question focusing on the attribute aspect of spatio-temporal patterns can be 'where and when has the specified patterns (e.g. with decreasing value of the attribute)?' It can be answered by observing, hovering and also doing the selection in the heatmap. By directly observing the variations of the attribute's value in the heatmap for the co-clustering results, the user can identify regions and intervals with specified patterns. In the cases that patterns are too complicated, i.e. cannot be directly identified by observation, the user can also hover over the heatmap, which shows the detailed information of the hovered record, e.g. value, to help the identification of the specified patterns. Then by selecting these regions and intervals of interest through clicking the two locations and timestamps in the axes of the heatmap, the 
user can identify the corresponding regions (where) and time intervals (when) highlighted in the map and timeline respectively. Moreover, if there are several regions and intervals with

the specified patterns, the user can always repeat clicking buttons for selecting and clearing to record all of them. As such, these interactions enable users to visually explore the coclustering results to examine if the results are satisfactory for their specific objective and dataset at hand.

\subsection{Implementation}

We used open-source technologies to build this web-based platform. Hosted on a web server set up using Python ${ }^{2}$, the server side of the platform was developed in $\mathrm{R}$ for its rich libraries while the client side was implemented using Data-Driven Documents ${ }^{3}$ (D3) for its powerful capabilities of visualizations. For the connections between the server and client sides, we used the jug library of $\mathrm{R}^{4}$ to set up a simple REST API in the server to accept POST requests from the client. Then it was also used to send back RESPONSE to the client side after the co-clustering processing. Finally, it is important to mention that all codes from both client and server sides of this platform are to be made open-source in GitHub.

\section{Case study and results}

\subsection{Case study dataset}

Located in the northwest of Europe (bottom right of Figure 4), the Netherlands borders with the North Sea in the west and north where the weather is more influenced by moderate maritime climate while in the south and east it is bordered by Belgium and Germany more influenced by continental climate. As a result, the temperatures in these areas are different in this country despite of its relatively small territory (ab. $\left.41,500 \mathrm{~km}^{2}\right)$.

\footnotetext{
2 https://www.python.org/

${ }^{3} \mathrm{https}: / / \mathrm{d} 3 \mathrm{js}$. org/

${ }^{4}$ http://bart6114.github.io/jug/
} 


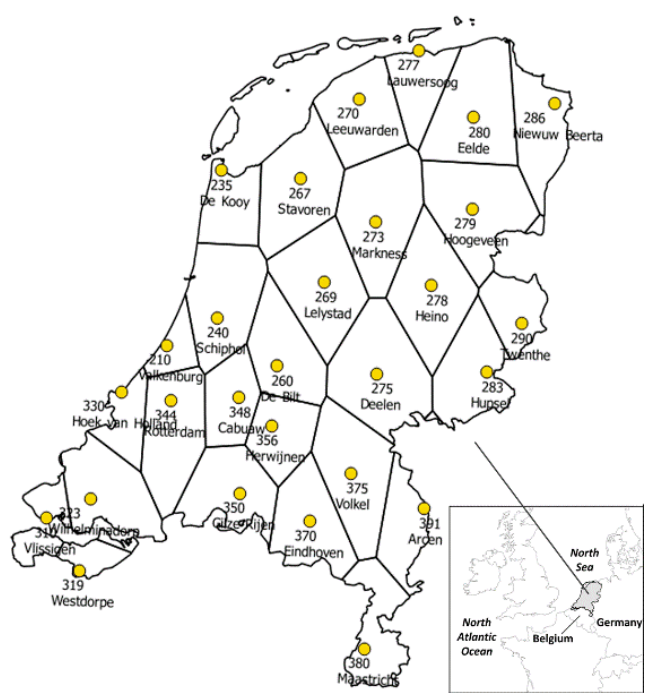

Figure 4: The Thiessen polygon map of 28 Dutch weather stations

More specifically, we use annual average temperature from 1992 to 2011 (20 years) at 28 weather stations, which are available from the Royal Netherlands Meteorological Institute ${ }^{5}$. A Thiessen polygon map was used to show the area under each station's influence (Figure 4).

\subsection{Visual exploration of the Dutch annual average temperature dataset}

After we upload the temperatures and Dutch Thiessen polygon map data, the platform provides both overview and details of the dataset, which helps users choose the parameters for BBAC_I, e.g. numbers of station- and year-clusters. For this temperature dataset, the color scheme $<$ RedYellowBlue $>$ is selected.

As showed in Figure 5, the platform provides an overview of the temperature dataset in its default view, i.e. before user interactions. The heatmap shows low temperature values in the year 1993, 1996 and 2010. Besides, it shows strange low values in 1998 at station 278 and 1999 at 267.

\footnotetext{
${ }^{5}$ www.knmi.nl
} 


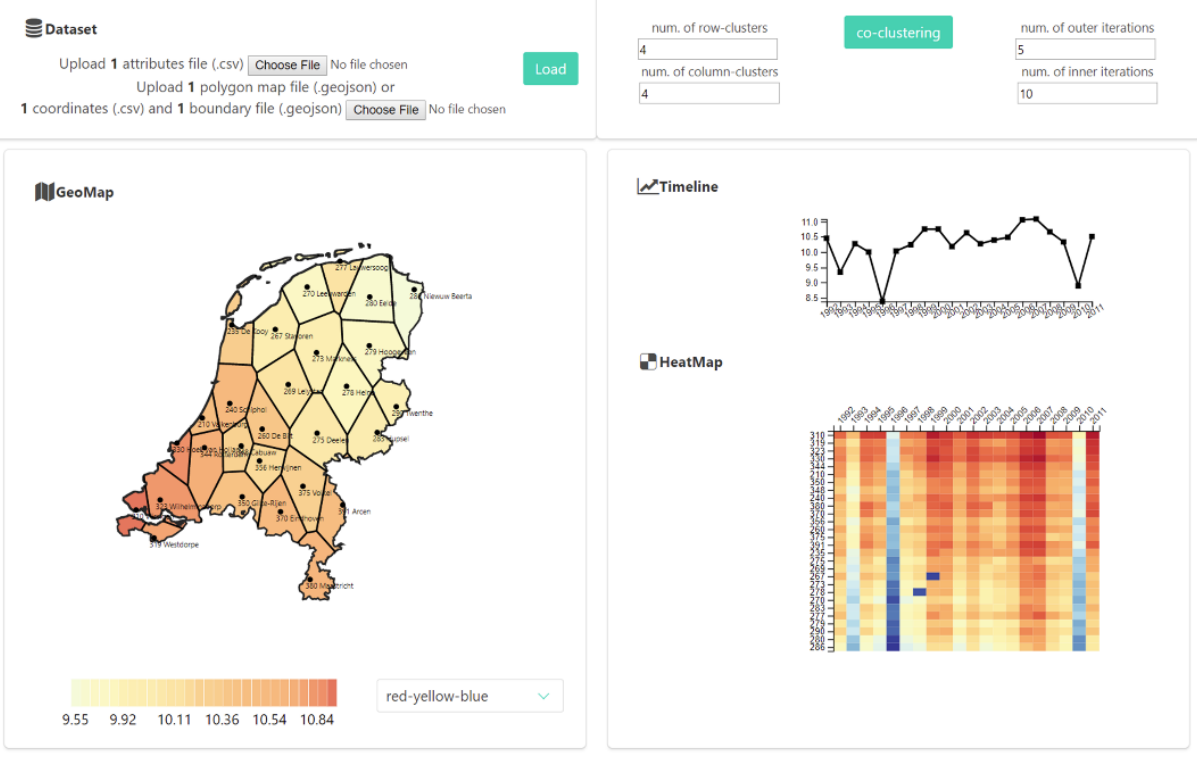

Figure 5: Overview of the Dutch yearly temperature dataset

By interacting with these linked visualizations, we can explore more details of the temperature dataset using co-clustering related tasks and questions. Example of questions for the spatial task can be "what is the temporal variation of temperature in station 290 (Twente) and which stations have similar variations in temperature?' To answer it, the user can hover over this station in the map and will see the similar increasing temperature over the study period and low temperatures in 1996 and 2010. With prior knowledge about the dataset (different temperatures in the southwest and northeast), the user can hover over other stations in the northeast to observe similar variations. These linked views, facilitating the selection of the number of station-clusters, suggest 4 as a reasonable number to indicate the transition of temperatures in the Netherlands.

Example of questions for the temporal task can be 'what is the spatial variation of temperature in 1996 and which years have similar variations in temperature?' It can be answered by hovering over 1996 in the timeline, the user will see the low temperature values over the whole study area in the map but still with increasing temperatures from southwest to northeast (left of Figure 6). By hovering over other years, the user will identify that 2010 has similar observations whereas 1993 is different in that only stations in the northeast have low temperatures 
(right of Figure 6). Thus, these observations suggest that years 1996 and 2010 should be in different year-clusters with 1993. Besides, these views suggest 2 other year-clusters with very high temperatures, e.g. 2006 and 2007 and with high ones, e.g. 2003 and 2004 (timelines and heatmaps in Figures 4, 5 and 6). Therefore, the number of year-clusters is suggested as 4 and this number also complies with human's common sense of temperatures for dividing years into "cold", "cool", "warm" and "hot" ones.

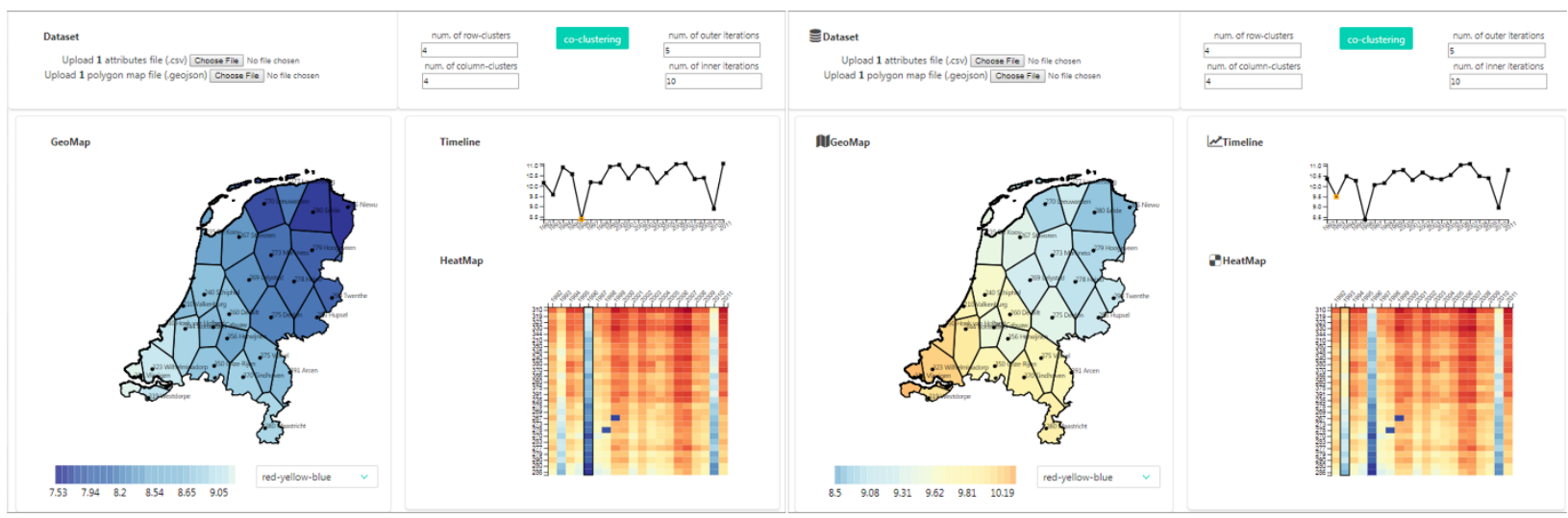

Figure 6: Detailed information of the dataset, 1996 (left) and 1993 (right) as examples

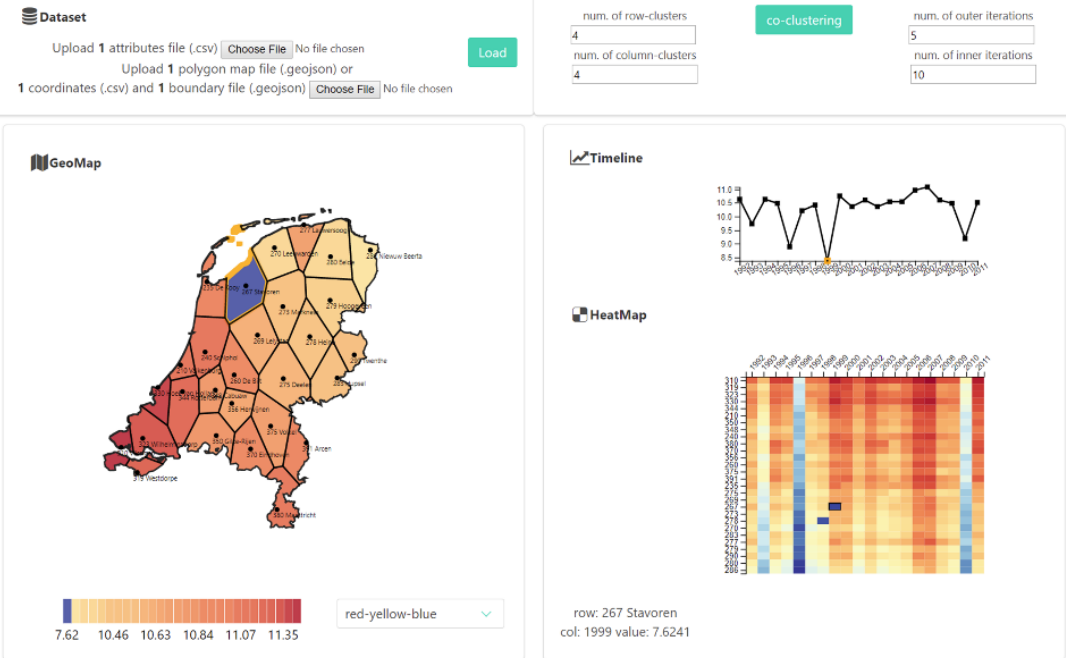

Figure 7: Detailed information of the dataset, the temperature in station "Stavoren" in 1999 as an example station 267 (Stavoren)?' To answer it, the user can hover over the temperature record in the 
heatmap for the station "Stavoren" in 1999 and detailed information, e.g. the temperature value etc. with the station and the year is highlighted in the map and timeline separately (Figure 7). As such, interactions with these views can help users to know more about this strange value or outlier in the dataset. Then users can make their own decisions for the next step, e.g. delete the outlier and re-upload or continue with the analysis. It is worth to mention that BBAC_I is insensitive to outliers [9] and therefore the user can continue the co-clustering analysis.

\subsection{Visual exploration of the co-clustering results}

With the numbers of station- and year-clusters suggested both as 4 in previous session and the number of inner and outer iterations as 1000 and 500 in previous study [9], the users can choose co-clustering parameters and click the co-clustering button in the interface. The co-clustering results of the temperature dataset are then displayed in the CMV (Figure 8).

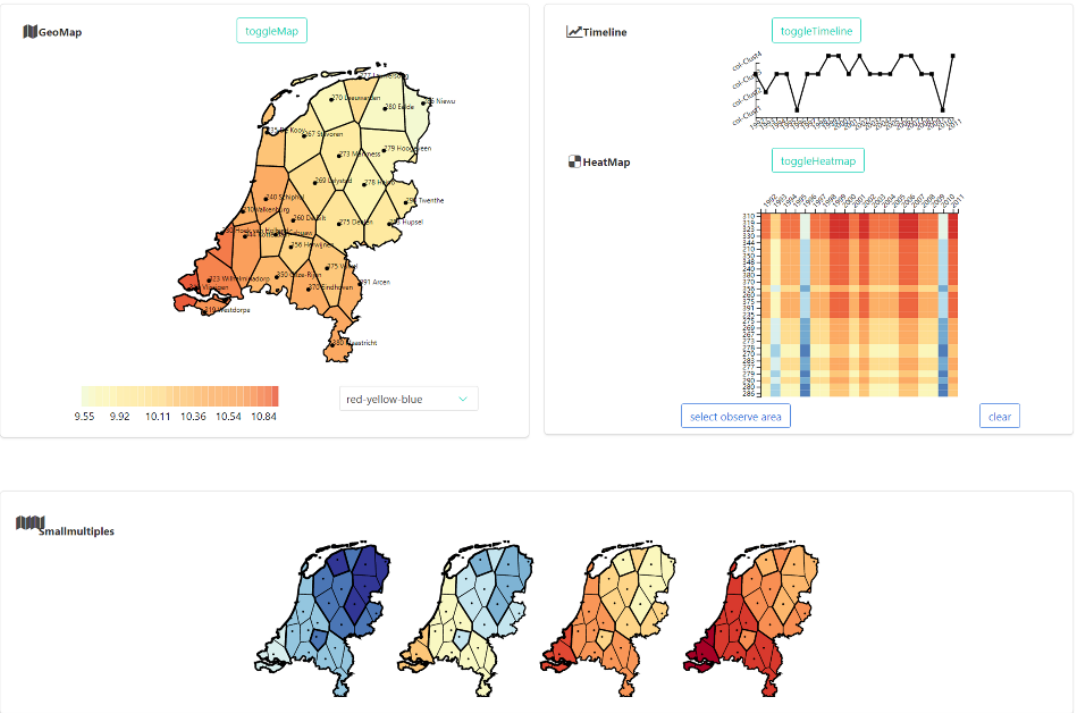

Figure 8: Co-clustering results of Dutch yearly temperature dataset temperature dataset using related tasks and questions. The example question for the spatial \& temporal aspect of individual co-clusters can be 'what is the co-cluster for station 280 (Eelde) in 1996 and which other stations and years are in the same co-cluster?' To answer it, the user can hover over the station 280 (Eelde) in the map and will see all stations belonging to station- 
cluster1 highlighted in the geographical map as well as all maps in the small multiples. Stationclusterl is in the northeast of the Netherlands with the lowest temperature over the whole country. Then the user can hover over 1996 in the timeline and see the highlighted first map in the small multiples for the year-cluster1 with the lowest temperature among all maps. Thus, the station 280 (Eelde) and 1996 belongs to the co-cluster which locates in the northeast of the country in the first map of small multiples with the lowest temperature. Finally, when the user hovers over this co-cluster, all stations and years that belong to this co-cluster will be highlighted in the geographical map and timeline respectively and the user can see other stations and years that are in the same co-cluster as the station 280 (Eelde) and 1996 (Figure 9).

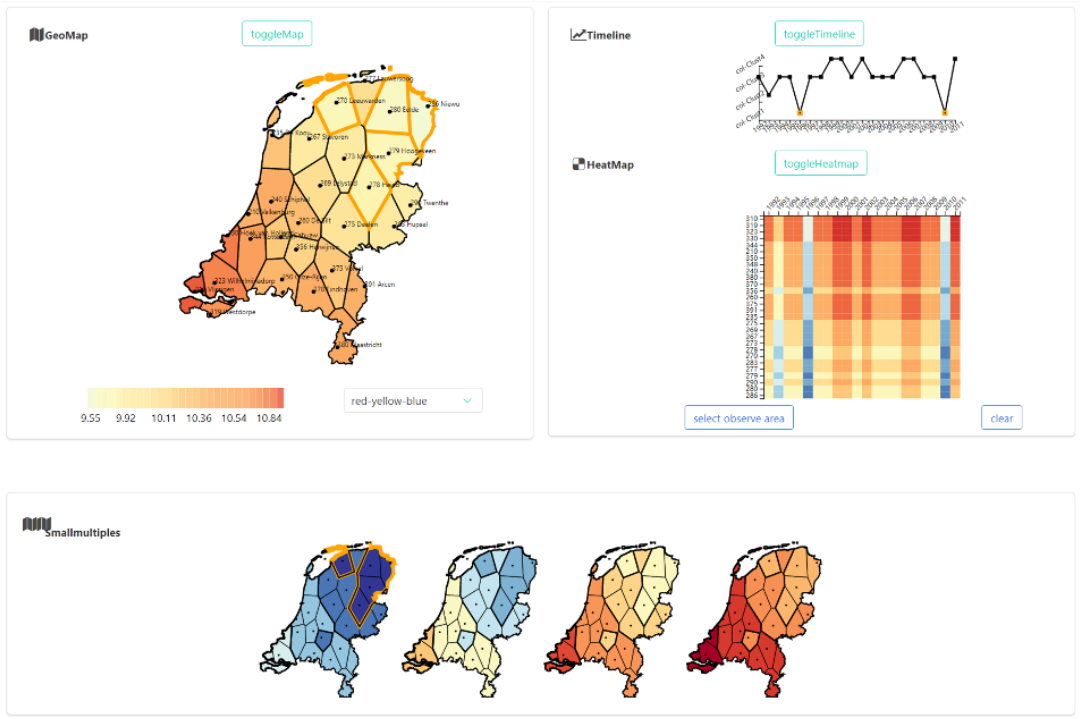

Figure 9: Interactions to explore the spatial \& temporal aspect of individual co-clusters, station 280 (Eelde) in 1996 as an example

The example question for the attribute aspect of individual co-clusters can be "what are stations and years in the co-cluster with the highest temperatures?' It can be answered by first observing all maps in the small multiples and identifying the co-cluster in the southwest of the country in the $4^{\text {th }}$ map as the one with the highest temperature. Then by hovering over this cocluster, the user can see the stations and years belonging to this co-cluster highlighted in the map and timeline separately. 


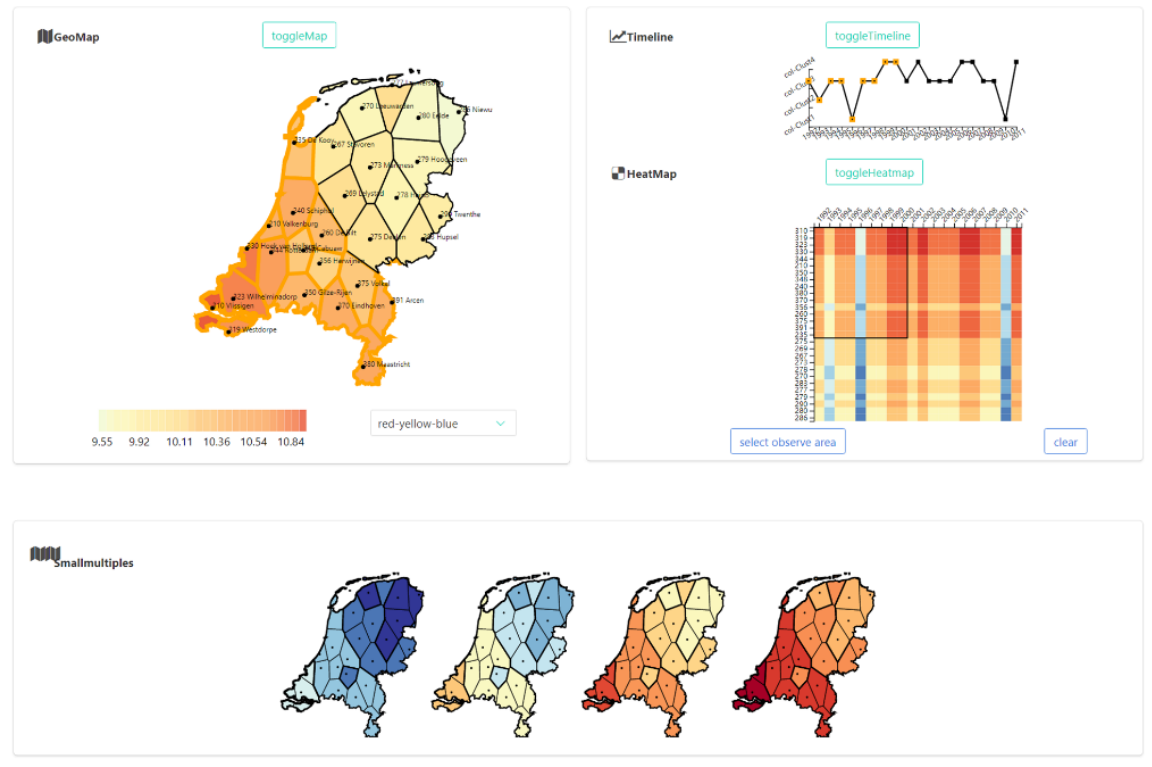

Figure 10: Interactions to explore the spatial $\&$ temporal aspect of patterns in the coclustering results, southwest half of the Netherlands from 1992 to 2000 as an example

The example question for the spatial \& temporal aspect of spatio-temporal patterns can be 'what are the temperature patterns in the southwest half of the Netherlands (including stations De Kooy, Schiphol, De Bilt, Volkel, Arcen and other stations of southwestwards) from 1992 to 2000?' To answer it, the user can click the station IDs 235 (De Kooy) and 310 (Vlissigen) in the y-axis of the heatmap to specify the southwest half region and also years 1992 and 2000 in the xaxis to specify the time period. Then after clicking the $<$ select observe area $>$ button, the user can observe that the temperatures within in the rectangle have a general increasing trend from regions in the center, i.e. nearby station De Kooy in earlier years, i.e. around 1992 to regions in the southwest, i.e. nearby station Vlissigen in recent years, i.e. around 2000 (Figure 10). Even with the general increasing trend, the user can also observe the decreasing temperature from station De Bilt (260) to station Herwijnen (356) in this period. Besides, the user can observe a decreasing trend of temperature from 1992 to 1993 and a sharp decreasing trend from 1995 to 1996 in the whole region. 
The example question for the attribute aspect of spatio-temporal patterns can be 'where and when has occurred the most increasing temperatures?' To answer it, the user can first observe the heatmap to identify $2010-2011$ as the period with the most increasing temperatures. Then for the regions, the user can hover over stations with different values of co-clusters to examine the

\section{Discussion}

414 After the first use of co-clustering in the geo-field, this topic attracted the attention of geographers 415 and the co-clustering methods have been applied in climatology, phenology and disease detection etc. [9-

416 11, 32]. For instance, Wu, Zurita-Milla [9] applied a co-clustering algorithm to analyze Dutch weather 417 patterns. Ullah, Daud [11] used a co-clustering algorithm to detect disease occurrences and Andreo, 418 Izquierdo-Verdiguier [32] applied the algorithm to identify potential spatial and temporal concurrence of 419 favorable conditions for West Nile virus outbreaks. The increasing number of applications of co420 clustering in the geo-field require an easily accessed platform with geovisual analytics functionality to

421 facilitate the analysis of spatio-temporal data, especially for non-experts. Compared with the 422 aforementioned studies, our web-based platform offers users a way to perform a visual and exploratory 423 co-clustering analysis with any web browser. Besides, interactions using CMV can help users to select 424 suitable sets of co-clustering parameters and can also facilitate the further interpretation of co-clustering 425 results. For instance, compared with the study of Wu, Zurita-Milla [9] which analyzed the same dataset 
using the same co-clustering algorithm and (geo)visualizations as in this study, our interactive platform

427 allows a better understanding of the data and co-clustering parameters (as illustrated in section 4.2).

428

429

430

431

432

433

434

435

436

437

438

439

440

441

442

443

444

445

446

447

448

449

450

Moreover, it is also important to mention that our platform is generic and has a strong potential to facilitate the analysis of spatio-temporal patterns in various other fields because it allows users to upload their own dataset. Take the analysis of population migration for example. [33] identified the natural communities/regions to represent the migration flows patterns by clustering locations to regions. We argue that since migration flows change over time, an interactive co-clustering analysis would reveal more underlying flow patterns that spread over both regions and time periods. Our platform can also be used to analyze both spatial and temporal behavior of public transportation passengers. Instead of separately clustering passengers based on their temporal activities and then presenting spatial characterization of clusters [34], our co-clustering method is capable of grouping passengers based on both their spatial and temporal activities and then providing spatio-temporal characterization. This platform also facilitates the pattern analysis by letting the user select a suitable color scheme for the uploaded dataset from various available ones.

Besides geo-field, our web-based geovisual analytics platform could be useful for analyzing and revealing more insights from data in other fields. Many studies have developed visual analytics platforms using co-clustering methods in different fields, especially gene expression. For example, Heinrich, Seifert [35] presented an interactive visual analytics platform based on heatmap and PCP to select co-clusters in gene expression data. Fiaux, Sun [36] presented a visual analytics tool using data mining algorithms and preview pane to analyze co-clusters in textual datasets. Besides, Wu and Kasif [37] and Zhou, Ma [38] developed interactive web-based platforms for co-clustering gene expression data to which users have easy access with any web browser. Compared with these platforms, our geovisual analytics platform not only provides the heatmap that is typically used for displaying co-clustering results. It also provides several other geovisualizations that could be helpful in revealing insights from the data as well as their coclustering results. Take gene expression data for example, the linear timeline could be used to arrange all 
451 expressions (conditions) in $\mathrm{x}$-axis and their values in the data or cluster membership in the co-clustering

452 results in the $y$-axis. By this means, it helps users to get a better understanding of variations of conditions

453 or condition-clusters and thus gain more insights into both the original data and co-clustering results.

\section{Conclusion}

Following the work of [9] who introduced a co-clustering algorithm (BBAC I) to geo-field, here we present an interactive web-based geovisual analytics platform for the exploratory co-clustering analysis of spatio-temporal data. With easy access in any web browser, the platform allows users to visually and interactively explore their spatio-temporal data as well as the co-clustering results. More specifically, consisting of a front-end GUI and back-end server, this platform allows users to upload data and explore it using interactive coordinated multiple views (CMV), then choose parameters and implement the coclustering algorithm, and finally explore the co-clustering results.

To illustrate our developed platform, we explored Dutch yearly temperature dataset for 28 stations and 20 years. Co-clustering related tasks and questions are used to facilitate the exploration in a systematic way. Compared with previous studies on co-clustering spatio-temporal data, interactions using CMV in the platform helps the selection of the most suitable set of co-clustering parameters of uploaded data and facilitates the further interpretation of co-clustering results. Besides, our platform could be helpful in pattern analysis for both geo-field and other different fields by integrating both the heatmap and several other geovisualizations. Thus, easily accessed with any web browser enhanced by Web 2.0 standards, our geovisual analytics platform enables the exploration of concurrent spatio-temporal patterns from large amounts of spatio-temporal data by combing both computer power and human interpretative capabilities. 


\section{References}

475 1. Robinson, A.C., et al., Geospatial big data and cartography: research challenges and opportunities for making maps that matter. International Journal of Cartography, 2017: p. 1-29.

477 2. Morse, P., A. Reading, and C. Lueg, Animated analysis of geoscientific datasets: An interactive graphical application. Computers \& Geosciences, 2017. 109: p. 87-94.

3. Wu, X., R. Zurita-Milla, and M.-J. Kraak, Visual discovery of synchronization in weather data at multiple temporal resolutions. The Cartographic Journal, 2013. 50(3): p. 247-256.

4. Liu, Q., M. Deng, and Y. Shi, Adaptive spatial clustering in the presence of obstacles and facilitators. Computers \& geosciences, 2013. 56: p. 104-118.

5. Cheng, T., et al., Spatiotemporal data mining, in Handbook of regional science. 2014, Springer. p. 1173-1193.

6. Banerjee, A., et al., A generalized maximum entropy approach to bregman co-clustering and matrix approximation. Journal of Machine Learning Research, 2007. 8(Aug): p. 1919-1986.

7. Cai, R., L. Lu, and A. Hanjalic, Co-clustering for auditory scene categorization. IEEE TRANSACTIONS ON MULTIMEDIA, 2008. 10(4): p. 596-606.

8. Dhillon, I.S., S. Mallela, and D.S. Modha. Information-theoretic co-clustering. in The 9th International Conference on Knowledge Discovery and Data Mining (KDD). 2003.

9. Wu, X.J., R. Zurita-Milla, and M.J. Kraak, Co-clustering geo-referenced time series: exploring spatio-temporal patterns in Dutch temperature data. International Journal of Geographical Information Science, 2015. 29(4): p. 624-642.

10. Wu, X., R. Zurita-Milla, and M.-J. Kraak, A novel analysis of spring phenological patterns over Europe based on co-clustering. Journal of Geophysical Research: Biogeosciences, 2016. 121: p. 1434-1448.

11. Ullah, S., et al., Detecting space-time disease clusters with arbitrary shapes and sizes using a coclustering approach. Geospatial health, 2017. 12(2).

12. Wu, X., et al., Triclustering Georeferenced Time Series for Analyzing Patterns of Intra-Annual Variability in Temperature. Annals of the American Association of Geographers, 2018. 108(1): p. 71-87.

13. Berkhin, P. A survey of clustering data mining techniques. in Grouping Multidimensional Data: Recent Advances in Clustering. 2006.

14. Andrienko, N. and G. Andrienko, Exploratory Analysis of Spatial and Temporal Data - A Systematic Approach. 2006, Berlin: Springer-Verlag.

15. Guo, D., Human-machine collabration for geographic knowledge discovery with highdimensional clustering, in The Pennsy/vania State University, Department of Geography. 2003.

16. van den Elzen, S. and J.J. van Wijk. Small multiples, large singles: A new approach for visual data exploration. in Computer Graphics Forum. 2013. Wiley Online Library.

17. Storme, T., B. Derudder, and S. Dörry, Introducing cluster heatmaps to explore city/firm interactions in world cities. Computers, Environment and Urban Systems, 2019. 76: p. 57-68.

18. Roberts, J.C. State of the art: Coordinated \& multiple views in exploratory visualization. in Coordinated and Multiple Views in Exploratory Visualization, 2007. CMV'07. Fifth International Conference on. 2007. IEEE.

19. Andrienko, G., et al., Space, time and visual analytics. International Journal of Geographical Information Science, 2010. 24(10): p. 1577-1600.

20. Guo, D. and W. Jiang, Understanding Spatiotemporal Patterns of Multiple Crime Types with a Geovisual Analytics Approach. 2013. 
21. Tatiana, V.L., et al., MobilityGraphs: Visual Analysis of Mass Mobility Dynamics via SpatioTemporal Graphs and Clustering. IEEE Transactions on Visualization \& Computer Graphics, 2016. 22(1): p. 11-20.

22. Delmelle, E.M., et al., A web-based geospatial toolkit for the monitoring of dengue fever. Applied Geography, 2014. 52: p. 144-152.

23. Barassi, V. and E. Treré, Does Web 3.0 come after Web 2.0? Deconstructing theoretical assumptions through practice. New media \& society, 2012. 14(8): p. 1269-1285.

24. Sun, G., et al., A Web-based visual analytics system for real estate data. Science China Information Sciences, 2013. 56(5): p. 1-13.

25. Sun, M., et al., A web-based geovisual analytical system for climate studies. Future Internet, 2012. 4(4): p. 1069-1085.

26. Liao, Z., et al. A web-based visual analytics system for air quality monitoring data. in Geoinformatics (Geolnformatics), 2014 22nd International Conference on. 2014. IEEE.

27. Banerjee, A., et al., A generalized maximum entropy approach to bregman co-clustering and matrix approximation. Journal of Machine Learning Research, 2007. 8: p. 1919-1986.

28. Silva, S., J. Madeira, and B.S. Santos. There is more to color scales than meets the eye: a review on the use of color in visualization. in Information Visualization, 2007. IV'07. 11th International Conference. 2007. IEEE.

29. Eisen, M.B., et al., Cluster analysis and display of genome-wide expression patterns. Proceedings of the National Academy of Sciences, 1998. 95(25): p. 14863-14868.

30. MacEachren, A., et al. Exploring high-D spaces with multiform matrices and small multiples. in Information Visualization, 2003. INFOVIS 2003. IEEE Symposium on. 2003. IEEE.

31. Andrienko, N. and G. Andrienko, Exploratory analysis of spatial and temporal data: a systematic approach. 2006: Springer Science \& Business Media.

32. Andreo, V., et al. Identifying Favorable Spatio-Temporal Conditions for West Nile Virus Outbreaks by Co-Clustering of Modis LST Indices Time Series. in IGARSS 2018-2018 IEEE International Geoscience and Remote Sensing Symposium. 2018. IEEE.

33. Guo, D., Flow mapping and multivariate visualization of large spatial interaction data. IEEE Transactions on Visualization and Computer Graphics, 2009. 15(6).

34. Briand, A.-S., et al., Analyzing year-to-year changes in public transport passenger behaviour using smart card data. Transportation Research Part C: Emerging Technologies, 2017. 79(Supplement C): p. 274-289.

35. Heinrich, J., et al. BiCluster viewer: a visualization tool for analyzing gene expression data. in International Symposium on Visual Computing. 2011. Springer.

36. Fiaux, P., et al., Bixplorer: Visual analytics with biclusters. Computer, 2013. 46(8): p. 90-94.

37. Wu, C.-J. and S. Kasif, GEMS: a web server for biclustering analysis of expression data. Nucleic acids research, 2005. 33(suppl_2): p. W596-W599.

38. Zhou, F., et al., QServer: a biclustering server for prediction and assessment of co-expressed gene clusters. PloS one, 2012. 7(3): p. e32660.

\section{Computer Code Availability}

The link of the GitHub repository for the source code used to develop the platform in this paper is https:/github.com/XWU-UT/Clustering_D3platform. We promise to make the repository public upon the acceptance of the manuscript. 